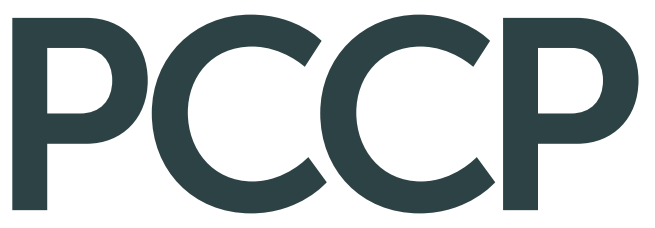

Physical Chemistry Chemical Physics www.rsc.org/pccp

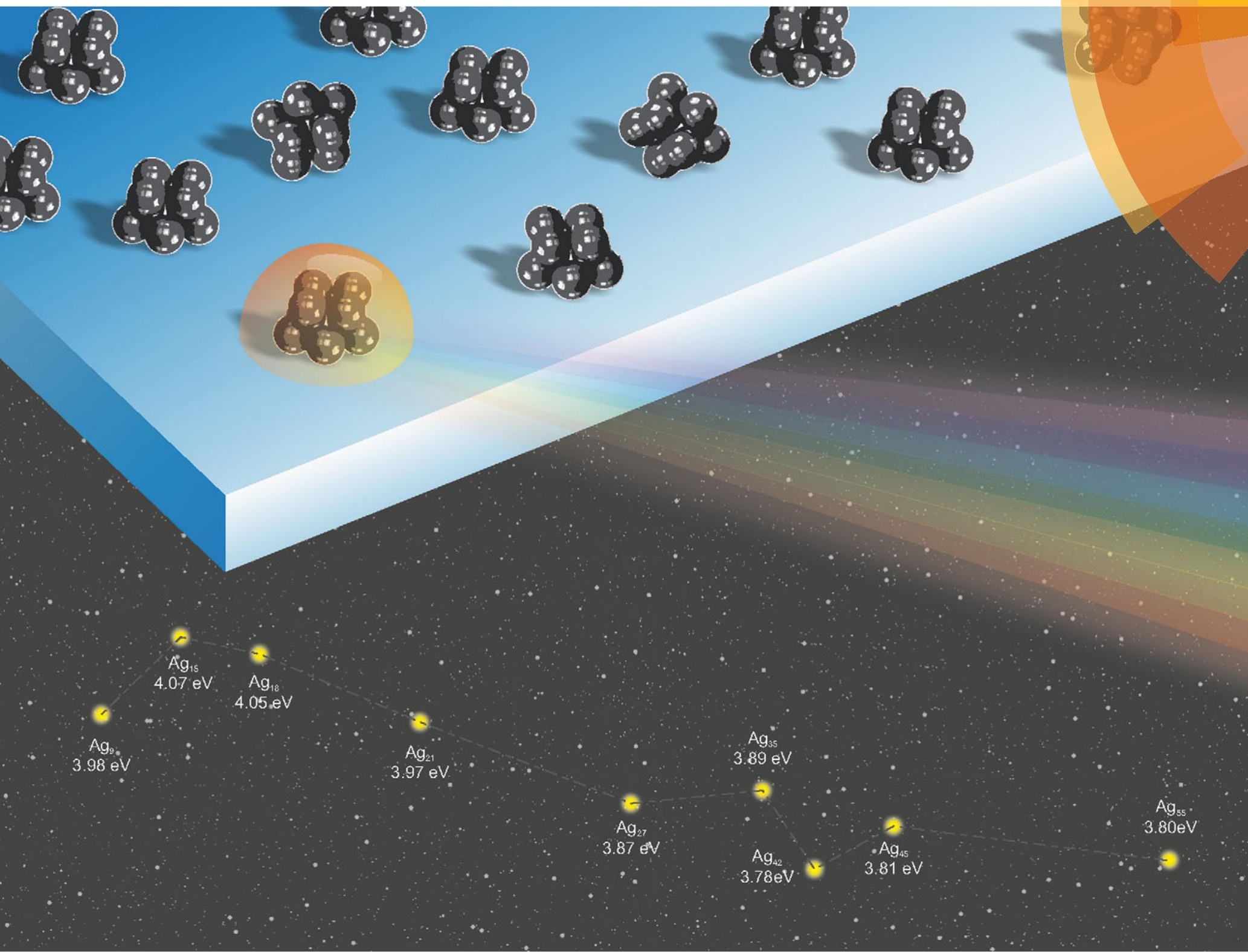

ISSN 1463-9076 


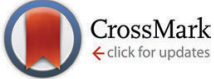

Cite this: Phys. Chem. Chem. Phys. 2015, 17, 17541

Received 18th March 2015, Accepted 12th May 2015

DOI: $10.1039 / \mathrm{c} 5 \mathrm{cp} 01582 \mathrm{k}$

www.rsc.org/pccp

\section{Plasmons in supported size-selected silver nanoclusters $\dagger$}

\author{
Tobias Lünskens, $\ddagger^{\mathrm{a}}$ Philipp Heister, $\ddagger^{\mathrm{a}}$ Martin Thämer, ${ }^{\mathrm{b}}$ Constantin A. Walenta, ${ }^{\mathrm{a}}$ \\ Aras Kartouzian ${ }^{* a}$ and Ulrich Heiz ${ }^{a}$
}

The plasmonic behavior of size-selected supported silver clusters is studied by surface second harmonic generation spectroscopy for the first time. A blue shift of $\sim 0.2 \mathrm{eV}$ in the plasmon resonance is observed with decreasing cluster size from $\mathrm{Ag}_{55}$ to $\mathrm{Ag}_{9}$. In addition to the general blue shift, a nonscalable size-dependence is also observed in plasmonic behavior of Ag nanoclusters, which is attributed to varying structural properties of the clusters. The results are in quantitative agreement with a hybrid theoretical model based on Mie theory and the existing DFT calculations.

Optical properties of metal nanoparticles (NPs) have been extensively studied in the past both theoretically and experimentally. Besides the fundamental scientific interest in understanding the behavior of material properties in the nanometer size regime and below, applications of metal NPs in light harvesting devices, photo-catalysis, sensors and optical materials have boosted the research in this field. ${ }^{1-5}$ Silver and gold are among the most studied NPs because of their pronounced plasmonic properties that depend on their size, shape, and chemical environment. ${ }^{6,7}$ Considerable progress in understanding the size-dependence of the plasmon resonance has been made over the past decades. Despite the great interest in the field that has triggered numerous studies on the gas phase ${ }^{8-10}$ and matrixembedded clusters down to the dimers, ${ }^{11-15}$ optical spectroscopic investigations on supported silver NPs, which are the most practically relevant systems for the applications mentioned above, have been limited to particle sizes above $1.5 \mathrm{~nm}$ in diameter. ${ }^{7,16-21}$ The main reasons underlying this shortcoming are the small surface coverages that are required to avoid agglomeration of particles in such samples, and the small extinction cross sections of silver nanoclusters, which push the conventional surface

\footnotetext{
${ }^{a}$ Technische Universität München, Department of Physical Chemistry, Catalysis Research Center, Lichtenbergstr. 4, 85748 Garching, Germany. E-mail: aras.kartouzian@mytum.de

${ }^{b}$ University of Chicago, 929 E. 57th Street, GCIS E 001A, Chicago, IL 60637, USA $\dagger$ Electronic supplementary information (ESI) available. See DOI: 10.1039/ c5cp01582k

\# These authors contributed equally to this work.
}

spectroscopic methods beyond their sensitivity limits. Consequently, a systematic experimental study of the optical behavior of supported $\mathrm{Ag}$ nanoclusters is still not available.

In order to overcome these challenges, single particle measurements have been performed in the past. Scholl et al. applied scanning transmission electron microscopy electron energy loss spectroscopy (STEM EELS) to study the plasmon resonances of individual silver NPs. Although they successfully circumvented the agglomeration issue, their smallest particle has a diameter of $1.7 \mathrm{~nm} .{ }^{22}$ In another approach, Schaffner $e t$ al. applied EELS to size-selected silver clusters consisting of up to 13 atoms supported on $\mathrm{MgO}$ thin films. ${ }^{23}$ The authors, however, did not comment on the position of the observed spectral features and limited their interpretation to a brief comparison with earlier reports. The results of Schaffner et al. seem not to be compatible with the literature except for a general resemblance to matrixembedded studies reported by Harbich et al. ${ }^{12}$

Recently, we demonstrated the strength of surface second harmonic generation (s-SHG) spectroscopy for investigation of optical properties of sub-monolayer molecular films and metal clusters. ${ }^{21,24}$ We reported on "plasmon splitting" in supported silver NPs with an average diameter of $1.5 \mathrm{~nm} .{ }^{21}$ In this work we employ s-SHG to study plasmonic properties of supported sizeselected silver clusters containing between 9 and 55 silver atoms. We succeeded for the first time in recording the optical response of such samples as a function of the precise number of atoms. Our results confirm the blue shift in the position of the plasmon resonance for silver nanoclusters with decreasing cluster size. Furthermore, the observed behavior can be explained using a hybrid theoretical model based on Mie theory and the size-dependent dielectric constants of the clusters reported in the literature. ${ }^{25}$ In addition to the general blue shift, these experiments reveal that size-dependent nonscalable variations of the optical response in Ag nanoclusters are observed, which pertain to shape of the clusters.

The cluster deposition facility, the spectroscopic arrangement of the s-SHG setup, and the details of data analysis have been reported in earlier contributions. ${ }^{21,24,26-28}$ Briefly, a high-frequency 


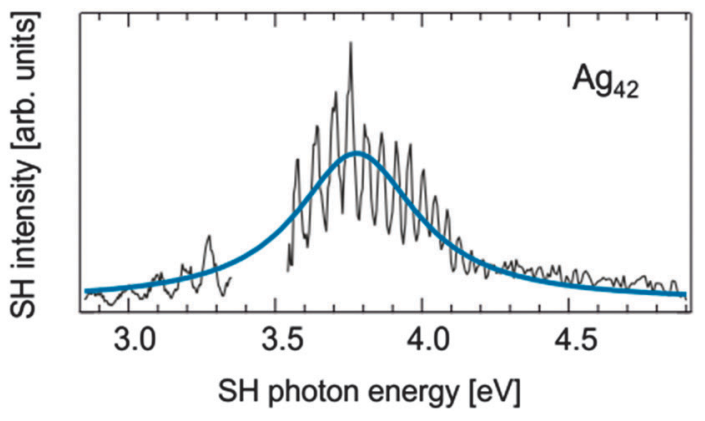

Fig. 1 s-SHG spectrum of size-selected $\mathrm{Ag}_{42}$ clusters on BK7 substrates showing a single broad resonance peak. The oscillations are due to the interference of the second harmonic beams generated at the two interfaces of the substrate and vacuum. These interferences can be precisely simulated and are removed from the data to achieve the original spectrum (solid line). The gap in the spectrum is due to the low laser intensity close to the signal-idler cross-over at $\sim 1.73 \mathrm{eV}(3.46 \mathrm{eV}$ of doubled light).

laser vaporization cluster source is used to generate a size distribution of metal clusters (cations, anions, and neutrals). A specific cluster size is then selected by passing the positively charged clusters through a quadrupole mass spectrometer with adequate mass resolution. Size-selected clusters are then softlanded onto BK7 substrates where they are neutralized by means of low-energy thermal electrons emitted from a hot filament. The spectroscopic studies were performed immediately after deposition under ultra-high vacuum conditions $\left(1 \times 10^{-10} \mathrm{mbar}\right)$ to avoid potential contamination of the samples. The surface coverage of the samples was held lower than $3 \times 10^{12}$ clusters per $\mathrm{cm}^{2}$ in order to prevent agglomeration and minimize cluster-cluster plasmon coupling (see S1, ESI $\dagger$ ).

Fig. 1 shows the recorded s-SHG spectrum of size-selected $\mathrm{Ag}_{42}$ clusters supported on BK7. The strong oscillation of the data points is caused by interferences between the SHG contributions from the two surfaces of the substrate. These interference effects owe to the nature of the spectroscopic method and are explicitly accounted for in the data analysis. ${ }^{26}$ The gap in the spectrum is caused by the lack of laser intensity close to the signal-idler cross-over at $710 \mathrm{~nm}$. The spectrum clearly indicates a single absorption band centered at $\sim 3.78 \mathrm{eV}$, which can be confidently assigned to the plasmon peak of silver.

In Fig. 2 normalized spectra of $\mathrm{Ag}_{n}$ cluster $(n=9,15,21,27$, $35,42,45$, and 55 ) on the BK7 glass substrate in the spectral range between $\sim 3$ and $\sim 5 \mathrm{eV}$ are depicted. For all clusters presented here, the spectra show a single peak. Earlier studies on size-selected $\mathrm{Ag}$ clusters embedded in rare gas matrices have shown that a single resonance peak dominates the optical spectra of clusters with more than 20 atoms. ${ }^{12-14}$ For smaller clusters it has been observed that a multiple peak structure emerges. ${ }^{29,30}$ Despite the immense fundamental importance of cryogenically cooled matrix-embedded clusters, they are of little practical use. In contrast, our samples are approximately at room temperature, are accessible for catalytic processes, and can be utilized as sensors. Due to the higher temperature of our samples, the possibly separated peaks cannot be resolved. Such a behavior has been observed for positively charged Ag clusters,

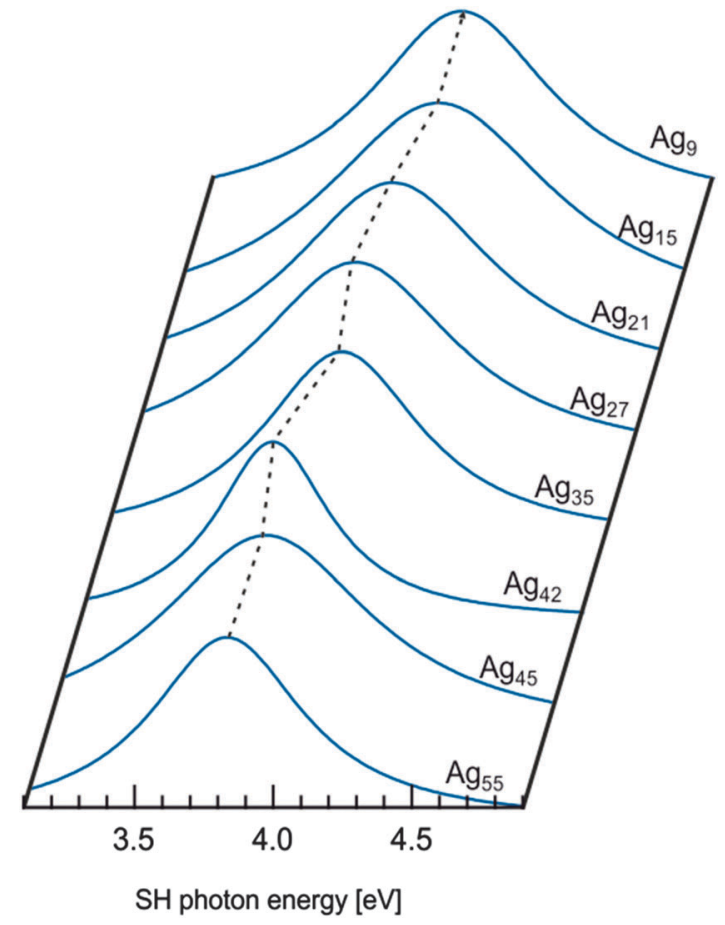

Fig. 2 The S-SHG spectra of size-selected $\operatorname{Ag}_{n}$ clusters $(9<n<55)$ on BK7. The dashed line connects the plasmon peak positions. For all the sizeselected Ag nanoclusters measured here a single resonance is observed. In general the position of the peak moves towards higher energies for smaller clusters, which can be described theoretically. The observed deviations from the general trend may be due to different geometries of the clusters.

where the information about the single peaks was not present due to the high temperature of the clusters. ${ }^{31}$ In the case of supported clusters, further line broadening may be caused by the existence of multiple isomers and various binding sites at the surface.

The dashed line in Fig. 2 links the positions of plasmon peaks for size-selected clusters measured here. As depicted more clearly in Fig. 3 an overall blue shift of the plasmon resonance with reducing cluster size is observed in agreement with the majority of literature. However, it should be noted that a red shift of plasmon resonance in small silver clusters has also been reported. ${ }^{12}$

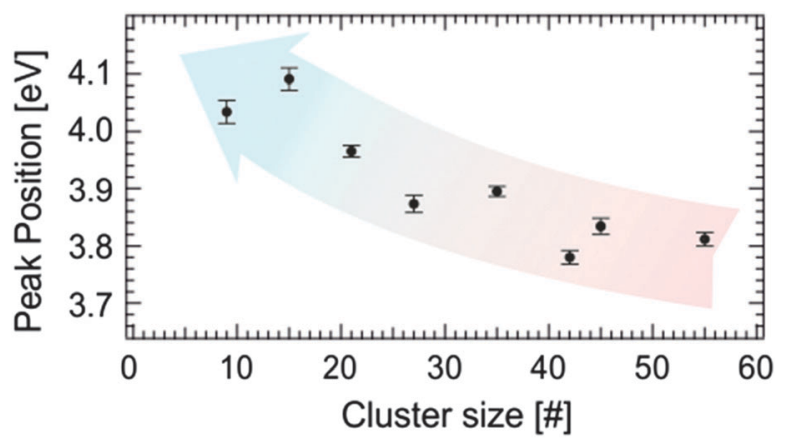

Fig. 3 The plasmon peak position plotted versus the number of atoms in the clusters. The plasmon resonance of supported size-selected silver nanoclusters shifts to higher energies for smaller clusters. 


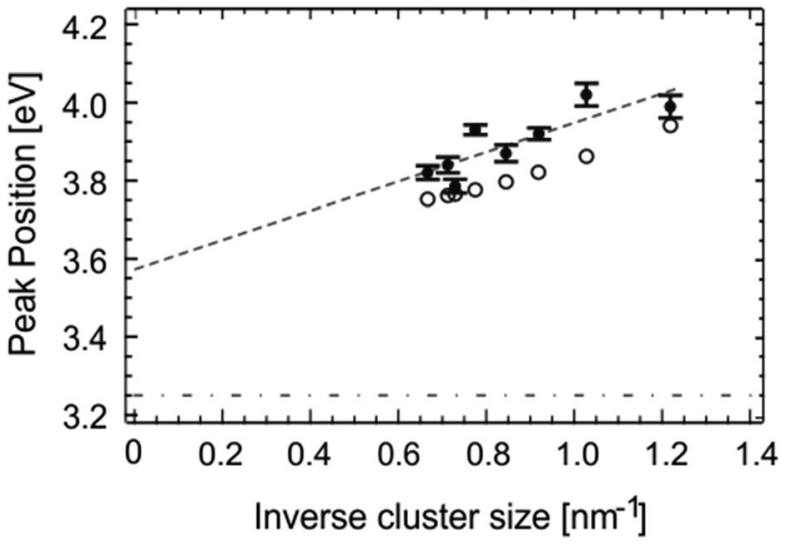

Fig. 4 The energy of the plasmon resonance of silver clusters plotted versus the inverse diameter of clusters (solid circles). The dashed-dotted line indicates the classical Mie model (size-independent). The dashed line results from considering the phenomenological effects of "spill-out" and "screening" of electrons. The open circles represent the theoretically predicted plasmon energies when the size-dependent dielectric functions are included.

We compared our experimental results with the following theoretical models: in the order of complexity, the classical Mie theory, the empirical model incorporating the phenomenological "spill-out" and "screen" effects, and a hybrid model including size-dependent dielectric function of the nanoclusters. Fig. 4 illustrates the experimentally observed plasmon peak positions as a function of inverse silver cluster diameters (see S2, ESI $\dagger$ ). Also shown in Fig. 4 are the corresponding results obtained from the theoretical models. Classical Mie theory has a long history in describing the optical properties of small metal NPs. In its original version, ${ }^{32}$ however, it is incapable of explaining a shift in the position of the plasmon peak when the particle size is varied. For spherical silver particles supported on the BK7 glass substrate, this model predicts a size-independent plasmon energy of $3.25 \mathrm{eV}$ (see S3, ESI $\dagger$ ) indicated by the horizontal dashed-dotted line in Fig. 4. It is important to note that the plasmon splitting effect, which is caused by non-spherical shape of NPs, is not sufficient to describe the blue shift observed here (see S3, ESI $\dagger$ ). The role of the nanoclusters' shape is considered later in the discussion.

The size-dependence of the plasmon frequency can be included in the Mie model qualitatively by introducing the so called "spill-out effect" as a correction in the dielectric function. In the case of silver as a $4 \mathrm{~d}$ metal the growing surface contribution with decreasing particle diameter results in an increasing amount of unscreened 5 s electrons, which leads to a change in the dielectric function causing the observed blue shift of the plasmon resonance. ${ }^{18,33}$ Such a classical extension has often been employed in the past with relative success in the description of the experimentally recorded spectra. This approach is, however, highly empirical and requires two rather unsupported assumptions: it assumes firstly, a single linear dependence of the plasmon energy on the inverse radius of the NPs for all particle sizes from dimers up to the bulk; and secondly, that the dielectric constants of the particles are unaltered regardless of their size. The inappositeness of the first assumption is readily revealed by reviewing various studies that address the size-dependence of the plasmon energy in $\mathrm{Ag}$ NPs, which demonstrates unambiguously that the shift of the plasmon resonance is more pronounced for larger NPs. ${ }^{18,31,34}$ In other words, the slope of a hypothetical line in a $1 / \mathrm{D}$ plot as in Fig. 4 is not constant over the whole range of particle sizes and is reduced for smaller clusters. It is therefore not surprising that extrapolating the linear fit to our data to the large particles limit $(1 / \mathrm{D}=0)$, indicated by the dashed line in Fig. 4 , results in an overestimation of plasmon energy. The inappropriateness of the second assumption is self-evident.

In the next level of the theory, the size-dependence of the dielectric functions needs to be considered explicitly as suggested by various groups in the past. ${ }^{22,35,36}$ He et al. used density functional theory to calculate the dielectric constants of a series of spherical silver clusters and achieved a sizedependent formalism. ${ }^{25}$ We adopted this approach and reproduced the plasmon energies of supported Ag nanoclusters. This model also predicts a blue shift of the plasmon energy with decreasing cluster size as shown by open circles in Fig. 4. In addition to the general trend, which confirms the experimental observations reported here, the predicted plasmon energies are also in a very good quantitative agreement with the experimentally measured values. A closer look at the data reveals that all of the experimentally measured data lie above the theoretical values suggesting that plasmon energies appear "bluer" than predicted. The value of this additional blue shift varies for different sizeselected nanoclusters. In order to describe this effect, we need to consider the selection rules of the employed spectroscopic method in more detail.

In s-SHG only transitions that possess a nonzero dipole moment perpendicular to the surface are probed. ${ }^{21,37}$ For spherical particles (as assumed in our models) plasmon resonances along different axes are identical. The plasmon resonance that will be measured by s-SHG (perpendicular to the surface) is not expected to deviate from the theoretically predicted value. Deviation of particle's shape from spherical form leads to variation of plasmon resonance energies along each of the three axes. This leads to splitting of the plasmon resonance in blue- and redshifted components for the short and long axes respectively. It was shown earlier, by combining s-SHG and surface cavity ring-down (s-CRD) spectroscopies, that silver clusters on BK7 substrates adopt an oblate shape. ${ }^{21}$ Consequently, for such clusters the plasmon resonance that can be observed in s-SHG is expected to be blueshifted as is the case for all the clusters measured here. The amount of the shift depends on the cluster's aspect ratio. For sizeselected clusters presented here s-CRD spectroscopy did not deliver a complementing signal, since the red-shifted component lies outside the available spectral range.

With the data presented in Fig. 4 we draw the following conclusions. For $\mathrm{Ag}_{9}, \mathrm{Ag}_{27}$ and $\mathrm{Ag}_{42}$ aspect ratios are closest to unity, since the observed peak positions are very close to the calculated values. For $\mathrm{Ag}_{15}, \mathrm{Ag}_{35}$, and $\mathrm{Ag}_{45}$ large blue shifts with respect to the calculated values are observed, which indicate an appreciable deviation from spherical shape. This observation suggests that $\mathrm{BK} 7$-supported $\mathrm{Ag}_{9}$ is a 3-dimensional cluster 
consisting of two atomic layers (e.g. 5 and 4 atoms) i.e. $\sim 2$ atoms high, $\sim 2$ atoms wide. Going to $\mathrm{Ag}_{15}$ the deviation drastically increases, indicating an increased aspect ratio for this cluster. This is compatible with the cluster being still 2 atomic layers high with more atoms per layer (e.g. 8 and 7 atoms) i.e. $\sim 2$ atoms high, $\sim 3$ atoms wide. For $\mathrm{Ag}_{21}$ the aspect ratio drops again and gets closer to the spherical limit, which indicates the emergence of the 3rd atomic layer. For $\mathrm{Ag}_{27}$ the aspect ratio is almost the same as for $\mathrm{Ag}_{21}$, i.e. $\sim 3$ atoms high, $\sim 3$ atoms wide, before increasing again for $\mathrm{Ag}_{35}$, where the height of the cluster is not changed but its width increases with the number of atoms. Following this analysis the next drop in the aspect ratio at $\mathrm{Ag}_{42}$ is caused by the emergence of the 4th atomic layer for BK7-supported silver clusters. Although direct confirmation of these effects may theoretically be delivered by atomic force microscopy (AFM), the inhomogeneity of BK7 surfaces renders an accurate determination of clusters' height impossible.

In summary, this work presents the first successful attempt on recording the size-dependent optical response of supported size-selected silver nanoclusters, which has been the topic of recent debate. ${ }^{38}$ Our results clearly show that the energy of the plasmon resonance in BK7-supported silver nanoclusters is blue-shifted with reducing cluster size. Using the hybrid theoretical model presented above, the experimental results could be described not only qualitatively but also quantitatively to a great extent. A more precise theoretical treatment may be given only by a fully quantum mechanical approach. On top of the general blue shift, we also found a nonscalable sizedependent plasmonic behaviour in supported Ag nanoclusters, which we attribute to the structural properties of the clusters. AFM studies on silver clusters supported on chemically equivalent and atomically flat surfaces such as $\mathrm{SiO}_{2}$ films grown on $\mathrm{Pt}(111)$ single crystals are planned for the future.

This work has been supported by BMBF through the project IC4, the European Research Council through an Advanced Research Grant (246645-ASC3), and the DFG through the project (HE 3454/21-1).

\section{Notes and references}

1 P. V. Kamat, J. Phys. Chem. B, 2002, 106, 7729-7744.

2 X. Chen, Z. F. Zheng, X. B. Ke, E. Jaatinen, T. F. Xie, D. J. Wang, C. Guo, J. C. Zhao and H. Y. Zhu, Green Chem., 2010, 12, 414-419.

3 S. Sarina, E. R. Waclawik and H. Zhu, Green Chem., 2013, 15, 1814-1833.

4 W. Hou, W. H. Hung, P. Pavaskar, A. Goeppert, M. Aykol and S. B. Cronin, ACS Catal., 2011, 1, 929-936.

5 P. Christopher, H. L. Xin and S. Linic, Nat. Chem., 2011, 3, 467-472.

6 K. L. Kelly, E. Coronado, L. L. Zhao and G. C. Schatz, J. Phys. Chem. B, 2002, 107, 668-677.

7 U. Kreibig, Optical Properties of Metal Clusters, Springer, 1995.

8 B. G. Deboer and G. D. Stein, Surf. Sci., 1981, 106, 84-94.
9 K. Laihing, P. Y. Cheng and M. A. Duncan, Z. Phys. D: At., Mol. Clusters, 1989, 13, 161-169.

10 L. D. Socaciu, J. Hagen, U. Heiz, T. M. Bernhardt, T. Leisner and L. Woste, Chem. Phys. Lett., 2001, 340, 282-288.

11 F. Conus, J. T. Lau, V. Rodrigues and C. Felix, Rev. Sci. Instrum., 2006, 77, 113103.

12 S. Fedrigo, W. Harbich and J. Buttet, Phys. Rev. B: Condens. Matter Mater. Phys., 1993, 47, 10706-10715.

13 W. Harbich, S. Fedrigo and J. Buttet, Chem. Phys. Lett., 1992, 195, 613-617.

14 W. Harbich, S. Fedrigo and J. Buttet, Z. Phys. D: At., Mol. Clusters, 1993, 26, 138-140.

15 I. Rabin, W. Schulze, G. Ertl, C. Felix, C. Sieber, W. Harbich and J. Buttet, Chem. Phys. Lett., 2000, 320, 59-64.

16 U. Kreibig and L. Genzel, Surf. Sci., 1985, 156, 678-700.

17 C. M. Grimaud, L. Šiller, M. Andersson and R. E. Palmer, Phys. Rev. B: Condens. Matter Mater. Phys., 1999, 59, 9874-9877.

18 N. Nilius, N. Ernst and H. J. Freund, Phys. Rev. Lett., 2000, 84, 3994-3997.

19 P. Christopher and S. Linic, ChemCatChem, 2010, 2, 78-83. 20 A. Hotzel, S. Mathies, D. E. Starr, A. Grujic and M. Wolf, Appl. Phys. B: Lasers Opt., 2011, 103, 171-187.

21 M. Thamer, A. Kartouzian, P. Heister, T. Lunskens, S. Gerlach and U. Heiz, Small, 2014, 10, 2340-2344.

22 J. A. Scholl, A. L. Koh and J. A. Dionne, Nature, 2012, 483, U421-U468.

23 M. H. Schaffner, F. Patthey and W. D. Schneider, Eur. Phys. J. D, 1999, 9, 609-612.

24 M. Thämer, A. Kartouzian, P. Heister, S. Gerlach, M. Tschurl, U. Boesl and U. Heiz, J. Phys. Chem. C, 2012, 116, 8642-8648. 25 Y. He and T. F. Zeng, J. Phys. Chem. C, 2010, 114, 18023-18030. 26 A. Kartouzian, P. Heister, M. Thaemer, S. Gerlach and U. Heiz, J. Opt. Soc. Am. B, 2013, 30, 541-548.

27 A. Kartouzian, J. Antonowicz, T. Lunskens, A. Lagogianni, P. Heister, G. Evangelakis and R. Felici, Mater. Express, 2014, 4, 228-234.

28 A. Kartouzian, M. Thaemer, T. Soini, J. Peter, P. Pitschi, S. Gilb and U. Heiz, J. Appl. Phys., 2008, 104, 124313.

29 M. Harb, F. Rabilloud, D. Simon, A. Rydlo, S. Lecoultre, F. Conus, V. Rodrigues and C. Felix, J. Chem. Phys., 2008, 129, 194108.

30 S. Lecoultre, A. Rydlo, J. Buttet, C. Felix, S. Gilb and W. Harbich, J. Chem. Phys., 2011, 134, 184504.

31 J. Tiggesbaumker, L. Koller, K. H. Meiwesbroer and A. Liebsch, Phys. Rev. A: At., Mol., Opt. Phys., 1993, 48, R1749-R1752.

32 G. Mie, Ann. Phys., 1908, 25, 377-445.

33 A. Liebsch, Phys. Rev. B: Condens. Matter Mater. Phys., 1993, 48, 11317-11328.

34 K. P. Charle, W. Schulze and B. Winter, Z. Phys. D: At., Mol. Clusters, 1989, 12, 471-475.

35 R. C. Monreal, T. J. Antosiewicz and S. P. Apell, New J. Phys., 2013, 15, 083044.

36 S. Raza, N. Stenger, S. Kadkhodazadeh, S. V. Fischer, N. Kostesha, A. P. Jauho, A. Burrows, M. Wubs and N. A. Mortensen, Nanophotonics, 2013, 2, 131-138.

37 Y. R. Shen, Annu. Rev. Phys. Chem., 1989, 40, 327-350.

38 H. Haberland, Nature, 2013, 494, E1-E2. 